\title{
THE FLOW TRANSITION IN A BUBBLE COLUMN
}

\author{
TOSHIRO MARUYAMA, SATOSHI YOSHIDA \\ AND TOKURO MIZUSHINA \\ Department of Chemical Engineering, \\ Kyoto University, Kyoto 606
}

\begin{abstract}
The flow transition from uniform bubbling regime to liquid circulation regime was studied in a vertical two-dimensional bubble column with no net liquid flow. A regular two-loop liquid circulation was observed to occur as a result of violent interactions of unsteady liquid circulation and large bubbles. A critical superficial gas velocity was defined as the velocity at the point of incipient regular circulation and was obtained from the gas velocity at maximum gas hold-up. Under a uniform stable gas distribution the critical gas velocity depends on the ungassed liquid height and is independent of sparger geometry, i.e., the diameter and pitch of holes. When the pressure drop through the sparger is insufficient or inlet gas is distributed in the narrow central region of the sparger, interactions of large-scale liquid motions and large bubbles in the bulk section is suppressed by depleting the growth of large bubbles at the base. This makes the critical gas velocity larger than that under the uniformly sparged condition.

A similar dependence of critical gas velocity on ungassed liquid height was confirmed with literature data for three-dimensional bubble columns.
\end{abstract}

\section{Introduction}

In the bubble column there exist a uniform bubbling regime at lower superficial gas velocity and a liquid circulation regime at higher superficial gas velocity. The drift flux model for homogeneous dispersion has been applied successfully by Nicklin ${ }^{10)}$ and Wallis ${ }^{14}$ to describe uniform bubble flow. The theory of liquid circulation meanwhile has been developed by Miyauchi and Shyu ${ }^{9}$, Hills $^{4}$ and Ueyama and Miyauchi $^{13}$ to predict turbulent liquid circulation. However, little has been written about the flow transition from uniform bubbling regime to liquid circulation regime.

In bubble columns having a sparger hole diameter of less than about $1 \mathrm{~mm}$, it is well known that a transition regime ${ }^{14)}$ exists over a wide range of superficial gas velocity. The existence of this regime is characterized by a significant increase of average gas hold-up of the bed in comparison with the liquid circulation regime. Aoyama et al. ${ }^{2)}$ reported that the value of gas hold-up depended on sparger geometry and that the liquid axial dispersion coefficient showed the minimum value in the transition regime. Anderson and Quinn ${ }^{13}$ discussed some features of flow transition in the presence of trace contamination. Kawagoe et al. $^{6)}$ correlated the superficial gas velocities at upper and lower bounds of the transition region as a function of liquid hold-up. Their results showed

\footnotetext{
Received January 9, 1981. Correspondence concerning this article should be addressed to T. Maruyama. S. Yoshida is now with Nippon Steel Co. Ltd. Tokai 464.
}

that these velocities were independent of sparger geometry and bubble column diameter. Lockett and Kirkpatrick $^{8}$ have quantitatively explained deviations from uniform bubbly flow at the lower bound of the transition region on the basis of liquid circulation and the presence of large bubbles. Yamashita and Inoue ${ }^{15}$ ) measured gas hold-up under nonuniform inlet gas distribution in both two- and three-dimensional bubble columns. They correlated the maximum value of gas hold-up with parameters of the gas distributor. Recently, Zahradnik and Kaštánek ${ }^{16)}$ and Sakata and Miyauchi $^{12)}$ showed that the region of transition flow was determined by the value of superficial gas velocity and geometrical parameters of the distributing plates.

In this paper, the flow transitions will be discussed through visual observation of bubble motion in vertical bubble columns with no net liquid flow. To facilitate observations of bubble motion, a two-dimensional bubble column was used instead of a conventional three-dimensional bubble column. A critical superficial gas velocity will be defined on the basis of flow observations and measured static-pressure distribution. Dependence of the critical value on bed height and the influence of distributor geometry will be discussed by using measured overall gas hold-up and reported data for the three-dimensional bubble column.

\section{Experiments}

A schematic of the experimental apparatus is shown in Fig. 1. The bubble column is two-dimensional and open to the atmosphere, with a rectangular cross section of $30 \mathrm{~cm} \times 1 \mathrm{~cm}$ and a height of $130 \mathrm{~cm}$. The 


\begin{tabular}{|c|c|c|c|}
\hline & $\begin{array}{c}\text { Table } 1 \\
d[\mathrm{~cm}]\end{array}$ & $\begin{array}{r}\text { Injection } \\
l[\mathrm{~cm}]\end{array}$ & odermic tube) \\
\hline L2 & 0.02 & 2.0 & \multirow{6}{*}{ 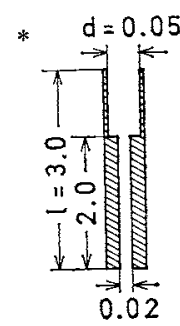 } \\
\hline S2 & 0.02 & 0.5 & \\
\hline L3 & 0.03 & 5.0 & \\
\hline S3 & 0.03 & 2.0 & \\
\hline L5* & 0.05 & 3.0 & \\
\hline S5 & 0.05 & 2.0 & \\
\hline
\end{tabular}

side walls are made of transparent acrylic resin. The gas distributor, made of stainless steel plate, has twenty-nine evenly spaced junctions of injection needles, projecting $10 \mathrm{~mm}$ above the base plate along the centre line. The junctions make the variation of hole size and distribution of air at the base very easy with the aid of various injection needles and blind caps. Table 1 summarizes the diameter $d$ and length $l$ of the injection needles. A perforated plate and a sintered porous plate were also used as gas distributors. The perforated plate was $5 \mathrm{~mm}$ in thickness and had 121 uniformly spaced holes of $0.2-\mathrm{mm}$ diameter. The sintered plastic plate was $10 \mathrm{~mm}$ in thickness with a mean pore diameter of $75 \mu \mathrm{m}$. The gas chamber was of $225 \mathrm{~cm}^{3}$ volume.

Tap water at room temperature was mainly used as the continuous phase in the experiments, but results were also obtained for $10 \mathrm{wt} \%$ glycerol-water solution and $0.075 \mathrm{wt} \%$ acetic acid solution. Gas was taken from the compressed air main. Oil in the air from the compressor was removed by a filter. The volumetric flow-rate of air was measured with the orifice meter and expressed as a superficial gas velocity at atmospheric pressure. During a run the superficial gas velocity was changed at constant hold-up of liquid phase. Runs were performed with superficial gas velocity up to about $25 \mathrm{~cm} / \mathrm{s}$ and the ungassed liquid level was varied from 20 to $80 \mathrm{~cm}$. The overall gassed liquid level was measured to determine the overall average value of gas hold-up (foam, if present, was not included). For measuring longitudinal distribution of static pressure head in the middle of the bubble stream, a pressure tap with a diameter of $1 \mathrm{~mm}$ was situated on the vertical centre line of the back face of the bed at $5-\mathrm{cm}$ height intervals. It was connected to a set of vertical manometers of glass tube filled with the column liquid. A still camera was used to photograph some typical flow patterns (shutter speed of $2 \mathrm{~s}$ ).

\section{Results and Discussion}

\section{1 Flow observation}

Figure 2 shows qualitative sketchs of observed flow pattern and gas hold-up as a function of superficial gas velocity. The extent of the transition regime is de-

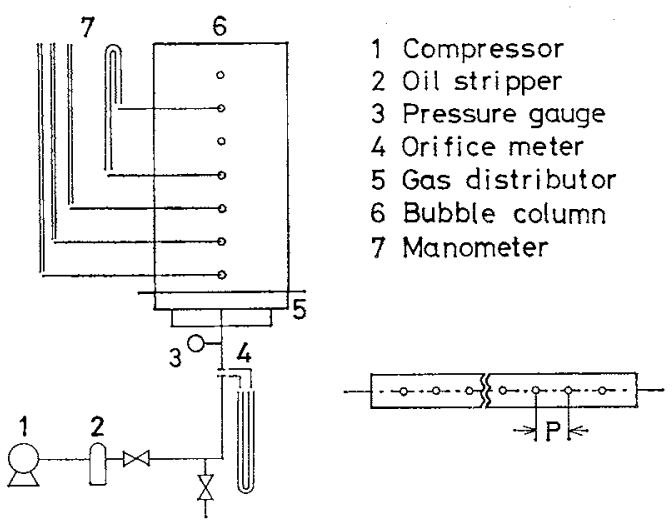

Fig. 1 Flow sheet of experimental apparatus and sketch of sparger hole distribution

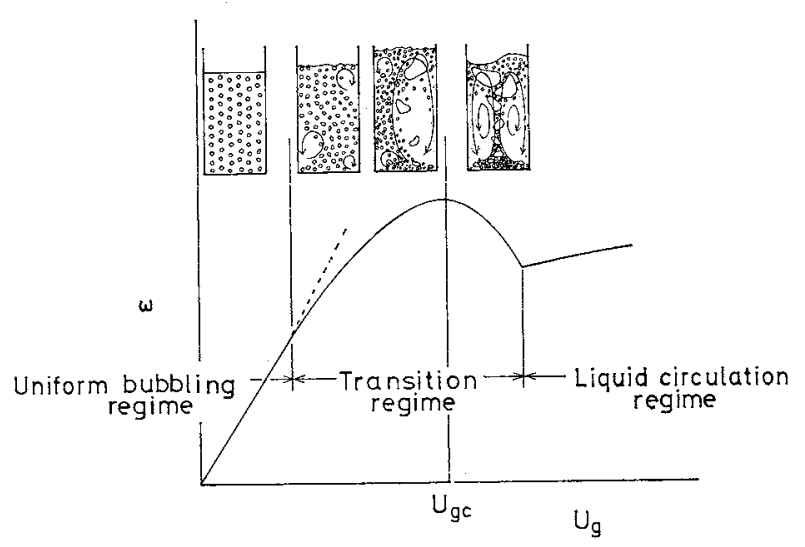

Fig. 2 Qualitative sketches of observed flow pattern and gas hold-up as a function of superficial gas velocity

fined after Wallis ${ }^{14}$.

At a low superficial velocity, the bubbles rise independently with fairly uniform spacing between them. The flow pattern then shows mostly random shortrange eddies. The lowest superficial gas velocity of transition regime corresponds to the point where overall gas hold-up $\varepsilon$ breaks away from the ideal curve for uniform bubbling. Observed change from uniform bubble flow to transition flow is asymptotic, depending on various factors ${ }^{7,8,14)}$ which affect the size of the gas bubble by altering the degree of coalescence. Under a coalescence-suppressing condition the most evident features observed are that the uniform bubble swarm begins to meander, indicating an occurrence of local liquid circulation near the side walls. With increasing $U_{g}$, bubble clusters begin to appear in the bulk of the column, and gross and local liquid circulations, i.e. turbulences in the liquid flow, develop randomly in space and time. Coalescence and breakup of bubbles can take place in the bubble cluster. There seems to be no regularity in it. Near the maximum gas hold-up, gross liquid circulation current sweeps the entire volume and the downward motion of gross liquid movement near the side wall affects the 

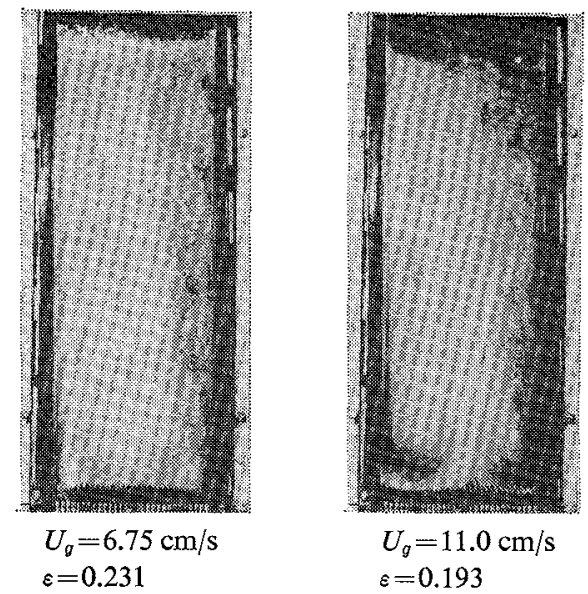

Fig. 3 Photographs of flow patterns at two different gas velocities $\left(H_{0}=65.0 \mathrm{~cm}\right)$

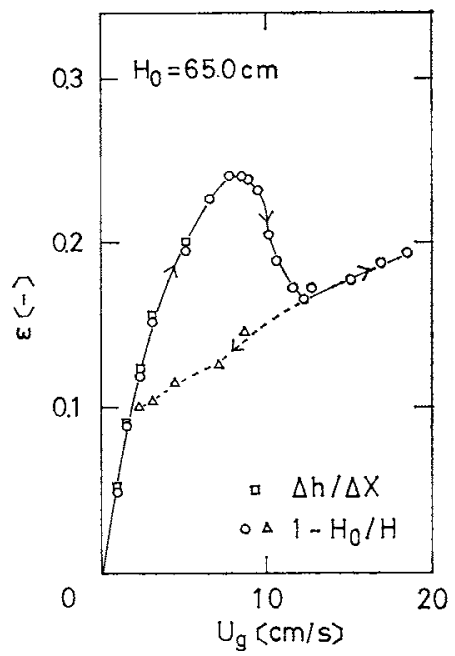

Fig. 4 Gas hold-up as a function of superficial gas velocity

steady bubble formation at the base. The downward flow suppresses the bubble rise in the side region, but continuous sparging of gas makes a large enough bubble to counteract the gross liquid circulation and damps it. Soon after that, another circulation commences to form in the other side of the column. With increasing $U_{g}$, the dynamic interaction of gross circulation and large bubble becomes frequent and violent. This makes a large periodical change in instantaneous gas hold-up.

At maximum gas hold-up, a symmetrical two-loop circulation upward in the middle and downward near the side walls appears for the first time among the violent and frequent interactions of large bubble and asymmetrical circulation. The photograph in Fig. 3 shows typical examples of the flow pattern with and without symmetrical liquid circulation. There are large eddies superimposed upon the liquid circulation.

The ordered flow pattern becomes precise at points to the right of the maximum on the curve. Large bubbles simultaneously appear on both sides of column

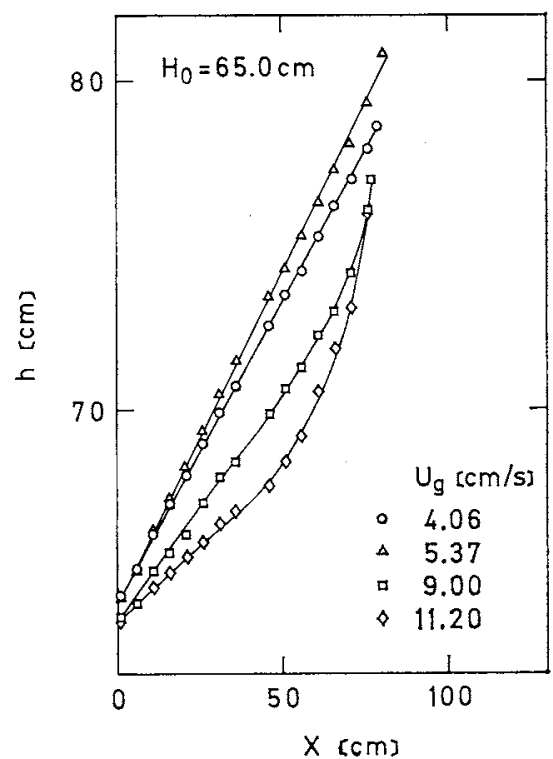

Fig. 5 Pressure head distribution in middle of bubble stream

and rise through the middle. At the upper limit of $U_{g}$ in the transition regime, the gas hold-up takes its minimum value followed by its gradual increase in the liquid circulation regime.

Thus the depletion of increase in $\varepsilon$ at lower $U_{g}$ is mainly due to the gross and local circulations (liquid turbulence) which affect the coalescence and breakup of bubbles and thus raise bubble velocity as suggested by recent investigators ${ }^{3,8,14}$. The decrease in gas hold-up at higher $U_{g}$, however, is attributable to the development of regular circulation of liquid ${ }^{3,13)}$ which is firmly established in the liquid circulation regime.

The superficial gas velocity at the point of incipient regular circulation was defined as a critical superficial gas velocity, $U_{g c}$, and was determined from $U_{g}$ at maximum gas hold-up.

\section{2 Flow characteristics}

Some characteristics of flow transition will be discussed on the basis of experiments with air bubbling through tap water with uniform gas inlet distribution: injection needle L2 (designation in Table 1) $P=1 \mathrm{~cm}$, and $n=29$.

Figure 4 shows a typical example of the overall gas hold-up $\varepsilon$ as a function of superficial gas velocity $U_{g}$. At $U_{g}<U_{g c}$, the value of $\varepsilon$ calculated from static pressure difference agreed well with those from an increase in the liquid levels. Arrows in this figure indicate the hysteresis of the behaviour; when $U_{g}$ is decreased after $\varepsilon$ takes the minimum value, $\varepsilon$ does not reproduce the original curve (and so the maximum value) but rather traces a lower curve. The range shown by the broken line corresponds closely to that of transition flow. The hysteresis indicates that, once developed, flow with ordered liquid circulation is more stable than flow without it. 
Figure 5 shows distributions of the static pressure head in the middle of the bubble stream. At $U_{g}>U_{g e}$ the distribution becomes a curved one, indicating a change in flow structure. In general, the pressure gradient of two-phase flow, $d p / d X$, can be expressed as the sum of gravitational, accelerational and frictional pressure drops:

$$
d p / d X=(d p / d X)_{\mathrm{grav}}+(d p / d X)_{\mathrm{acc}}+(d p / d X)_{\mathrm{fric}}
$$

The overall pressure difference in Fig. 5 shows that the friction loss term is very small compared to the other two terms. In addition, according to the local hold-up measurements of previous investigators ${ }^{5 /}$ using a conductivity probe technique, it is assumed that there is no longitudinal hold-up variation except very near the bottom and upper surface of the bed. That is, $(d p / d X)_{\mathrm{grar}}$ is constant. Then, linear change of pressure gradient at $U_{g}<U_{g c}$ shows that the second term on the right-hand side of Eq. (1) can be neglected and consequently that the gas hold-up can be measured by the static pressure difference as shown in Fig. 4. Deviations from the linear change at $U_{g} \geqq U_{g c}$ meanwhile are attributable to a contribution of the second term in Eq. (1). Figure 6 compares the pressure gradient with the first term in Eq.(1), under the assumption that there is no variation of gas hold-up along the axis. The pressure gradient shows the deceleration region $\left(-d p / d X<-(d p / d X)_{\mathrm{grav}}\right)$ in the upper part of the column and the acceleration region $(-d p / d X>$ $\left.-(d p / d X)_{\text {grav }}\right)$ which progressively descends to the lower part of the bed with increasing $U_{g}$. It was thus confirmed that a symmetrical two-loop circulation developed at $U_{g} \geqq U_{g c}$.

\section{3 Critical superficial gas velocity}

Figure 7 shows effects of impurities on gas hold-up. Experiments (Runs 1-3) were repeated continuously without using a filter or changing the water. Quite different values of gas hold-up were obtained in the same apparatus. This may be due to the fact that the accumulation of trace quantity of impurity, such as compressor oil in water, alters the fluid purity and hence resistance to agglomeration. However, it is noted that maximum gas hold-up occurs at about the same gas velocity. This fact was confirmed by comparing results with those for glycerol solution and acetic acid solution; the latter is a liquid having sufficient surface activity to prevent coalescence. The results compared with those of water are shown in Fig. 7 and Fig. 8. Indeed, a comparison indicates the same value of $U_{g}$ at maximum gas hold-up, showing the profound effect of liquid properties on gas hold-up. Thus the value of $U_{g c}$ is independent of gas hold-up but it depends on initial bed height $H_{0}$. In Fig. 9, the value of $U_{g c}$ is plotted as a function of $H_{0}$. As shown by solid lines, it is inversely proportional to

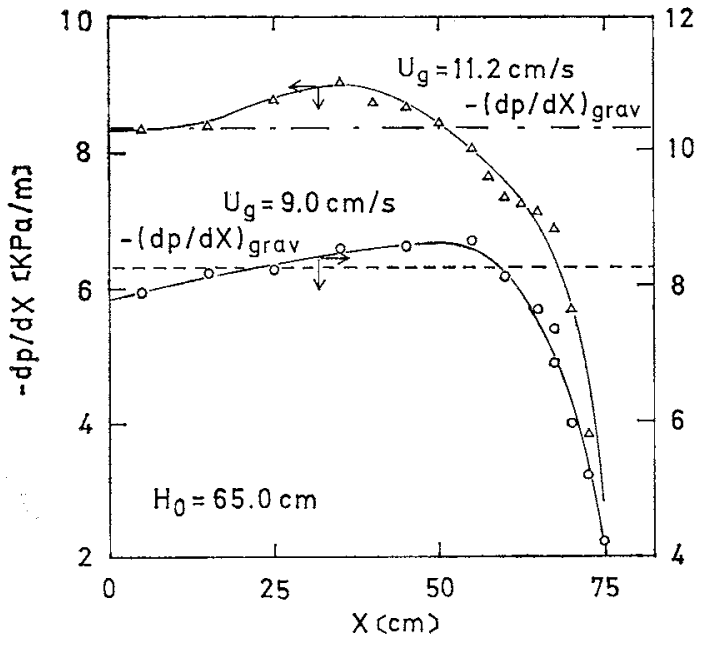

Fig. 6 Pressure gradient in middle of bubble stream

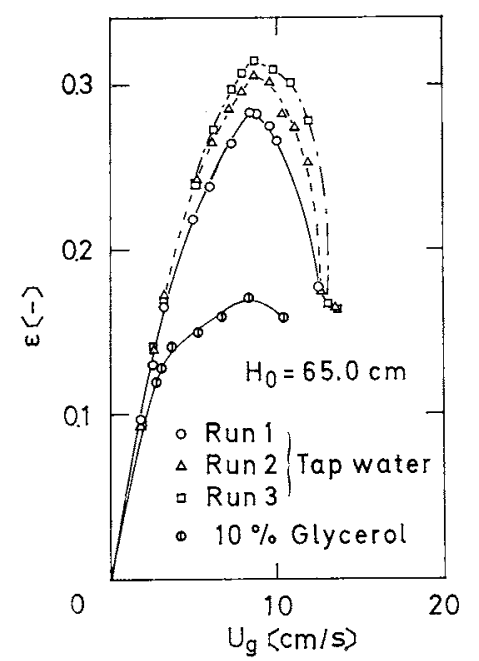

Fig. 7 Gas hold-up in water and glycerol solution

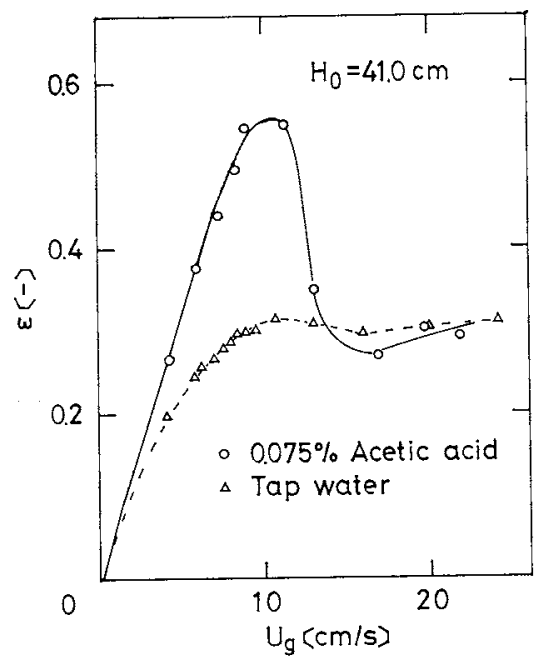

Fig. 8 Gas hold-up in water and acetic acid solution

$\sqrt{H_{0}}$, that is, $U_{g c}^{2} H_{0}$ is constant.

The value of $U_{g c}$ for the three-dimensional bubble column was obtained from the plot $\varepsilon$ vs. $U_{g}$ of some investigators. With experiments ${ }^{2}$ at a constant bed 


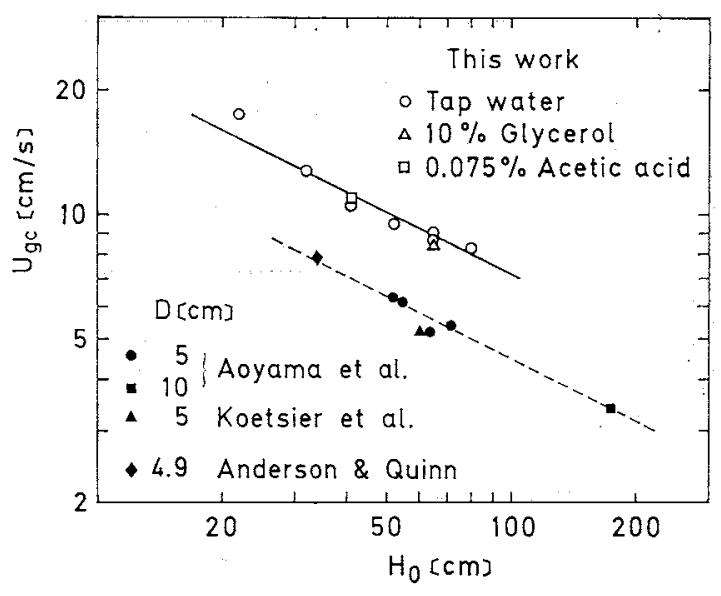

Fig. 9 Critical superficial gas velocity for twoand three-dimensional bubble columns

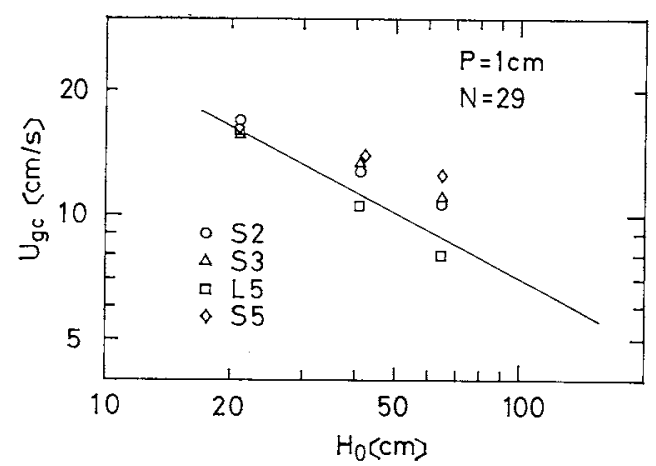

Fig. 10 Critical superficial gas velocity for various distributor hole sizes

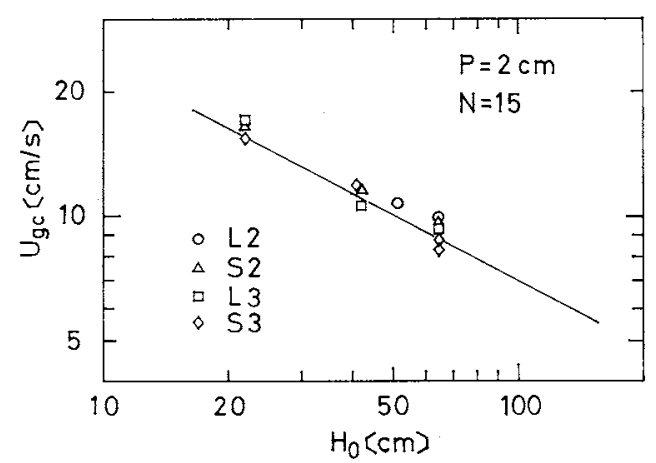

Fig. 11 Critical superficial gas velocity for $2-\mathrm{cm}$ pitch of distributor holes

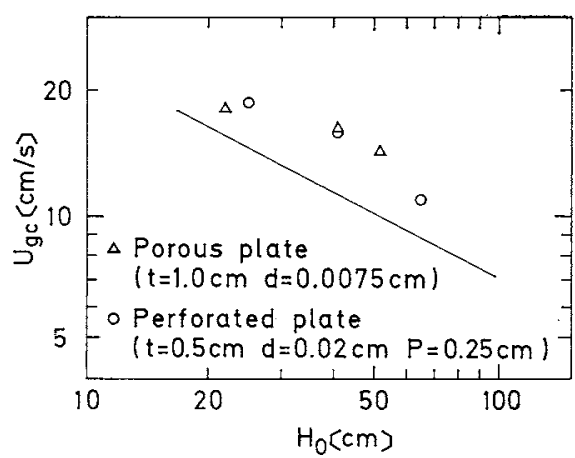

Fig. 12 Critical superficial velocity for perforated- and porous-plate spargers height $H$, the height of bed with no gas flow, $H_{0}$, was calculated from the value of $H$ and $\varepsilon$ at $U_{g c}$. All available data ${ }^{1,2,7)}$ for bubble column with porousplate sparger, which gives a uniform gas inlet distribution over the entire cross-section, show a consistent trend. The results are shown in Fig. 9 together with the correlation for two-dimensional bubble column. Although $U_{g e}$ of the latter is about two-thirds that of the former, a similar functional relationship against $H_{0}$ strongly suggests applicability of the preceding consideration to the more usual three-dimensional columns.

\section{4 Influence of distributor geometry}

Some experimental results for different diameters of injection needle are shown in Fig. 10. The value of $U_{g c}$ is apparently affected by distributor hole size. However, results with an injection needle L5 and those of $P=2 \mathrm{~cm}$ in Fig. 11 indicate that it is not attributable to hole size but rather to an insufficient pressure drop of gas in its passage through the hole.

In experiments under a small pressure drop of gas through the sparger, it was observed that the gas did not sparge stably and uniformly from evenly distributed holes. The change in the inlet gas flow according to unsteady gross liquid circulation postpones the appearance of large bubbles in both sides of the column and this is reflected by the persistence of unsteady liquid circulation. Hence the formation of steady two-loop liquid circulation, as a result of the strongly interacting large-scale motion of liquid and gas, is delayed to a larger superficial gas velocity. This was further confirmed with experiments using more uniformly distributed spargers with an insufficient pressure drop. The results for a perforated plate and a porous plate are shown in Fig. 12.

Thus the value of $U_{g c}$ is independent of the diameter and pitch of the hole under uniformly distributed inlet conditions. A sufficient pressure drop to ensure constant flow irrespective of liquid motion was about $70 \mathrm{kPa}$ and was independent of bed height. The following are results when the pressure drop is larger than $70 \mathrm{kPa}$.

Figures 13 and 14 show results under nonuniform inlet gas distribution. Designation (A) denotes asymmetrical partial (unilateral) distributions; (B) and (C) denote symmetrical partial distributions in border and central part, respectively. It is noted that the value of $U_{g c}$ is not affected by uneven inlet gas distributions except in the case of symmetrical partial distribution in the central part. Observations of bubble motion show that uneven inlet distributions do not cause partial aeration ${ }^{3)}$ above the distributor except at a very low gas rate. With increasing gas rate $\left(U_{g}<U_{g c}\right)$, liquid circulations induced directly by uneven inlet distribution become smaller in scale and confine them- 
selves in small region just above the nonaerated part of the distributor. This makes the bed above there uniformly aerated, similar to the case of uniform inlet gas distribution. On the other hand, the larger value of $U_{g c}$ under the partial distribution in the central region is attributable to a suppression of the growth of large bubbles on both sides of the column.

For the three-dimensional bubble column, the literature data ${ }^{2,3,11,12,15,16)}$ show that the value of $U_{g c}$ with perforated-plate sparger is larger than that with porous-plate sparger. From the above results for the two-dimensional column it is inferred that the larger values of $U_{g c}$ are mainly due to the deficits in uniform stable gas distribution: uneven inlet gas distribution or insufficient pressure drop of gas through the sparger.

\section{Conclusion}

At maximum gas hold-up, a regular two-loop liquid circulation appears first as a result of strong interactions of gross liquid circulations and large bubbles. A further increase in superficial gas velocity develops regular circulation to establish the liquid circulation regime.

The critical superficial gas velocity is defined as the velocity at the point of incipient regular circulation. It is obtained from the gas velocity at maximum gas hold-up. Under a uniform stable gas distribution the critical gas velocity depends on ungassed liquid height and is independent of sparger geometry, i.e. the diameter and pitch of holes.

A partial inlet gas distribution or an insufficient pressure drop of gas through the sparger decreases interactions of large-scale motions of liquid turbulence and large bubbles in the bulk section by depleting the growth of large bubbles at the base. Under these conditions, the critical superficial gas velocity becomes larger than that under a uniformly sparged condition.

A similar dependence of critical gas velocity on ungassed liquid height is applicable to the more usual three-dimensional columns. This fact strongly suggests a similarity in flow transitions between two- and three-dimensional columns.

\section{Nomenclature}

$\begin{array}{ll}D & =\text { inner diameter of three-dimensional } \\ & \text { bubble column } \\ d & =\text { inner diameter of injection needle } \\ H, H_{0} & =\text { gassed and ungassed liquid level from tip } \\ & \text { of injection needle, respectively } \\ h & =\text { pressure head based on } X=0 \\ l & =\text { length of injection needle } \\ N & =\text { number of injection needles } \\ P & =\text { pitch of injection needles } \\ p & =\text { static pressure } \\ t & =\text { thickness of sparger plate } \\ U_{g} & =\text { superficial gas velocity }\end{array}$

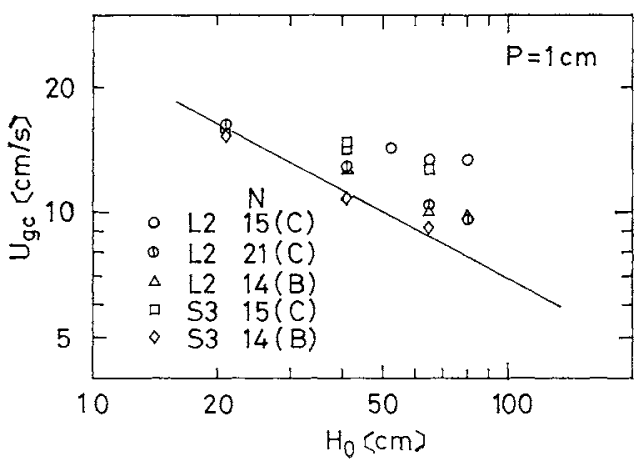

Fig. 13 Critical superficial gas velocity for symmetrical partial inlet gas distribution

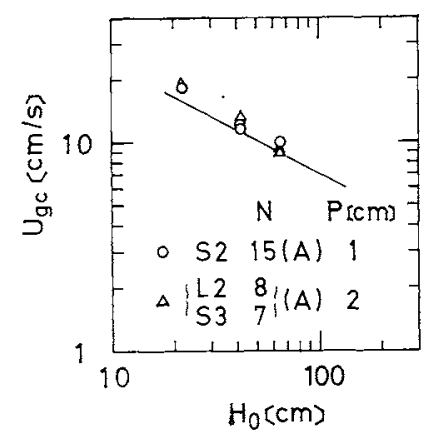

Fig. 14 Critical superficial gas velocity for asymmetrical inlet gas distribution

$$
\begin{aligned}
& U_{g c} \\
& X
\end{aligned}
$$$$
=\text { critical value of } U_{g}
$$

$[\mathrm{cm} / \mathrm{s}]$

$=$ longitudinal distance from tip of injection needle

[cm]

$=$ fractional gas hold-up

\section{Literature Cited}

1) Anderson, J. L. and J. A. Quinn: Chem. Eng. Sci., 25, 373 (1970).

2) Aoyama, Y., K. Ogushi, K. Koide and H. Kubota: $J$. Chem. Eng. Japan, 1, 158 (1968).

3) Freedman, W. and J. F. Davidson: Trans. Instn. Chem. Engrs., 47, T251 (1969).

4) Hills, J. H.: ibid., 52, 1 (1974).

5) Kato, Y., M. Nishinaka and S. Morooka: Kagaku Kogaku Ronbunshu, 1, 530 (1975).

6) Kawagoe, M., T. Inoue, K. Nakao and T. Otake: Kagaku Kógaku, 38, 733 (1974).

7) Koetsier, W. T., W. P. M. Van Swaaij and M. Van Der Most: J. Chem. Eng. Japan, 9, 332 (1976).

8) Lockett, M. J. and R. D. Kirkpatrick: Trans. Instn. Chem. Engrs., 53, 267 (1975).

9) Miyauchi, T. and C. N. Shyu: Kagaku Kögaku, 34, 958 (1970).

10) Nicklin, D. J.: Chem. Eng. Sci., 17, 693 (1962).

11) Ohki, Y. and H. Inoue: ibid., 25, 1 (1970).

12) Sakata, M. and T. Miyauchi: Kagaku Kogaku Ronbunshu, 6, 428 (1980).

13) Ueyama, K. and T. Miyauchi: AIChE J., 25, 258 (1979).

14) Wallis, G. B.: "One-Dimensional Two-Phase Flow", McGraw-Hill, New York (1969).

15) Yamashita, F. and H. Inoue: J. Chem. Eng. Japan, 8, 334 (1975).

16) Zahradnik, J. and F. Kaštánek: Chem. Eng. Commun., 3, 413 (1979). 